\title{
Johann Peter Frank (1745 - 1821). Wanderer zwischen den Welten, Wegbereiter der Sozialmedizin und Dermatologie
}

\author{
Johann Peter Frank (1745 - 1821). Migrant through Europe, Founder of Social Medicine, Public Health, \\ with an Impact on Dermatology
}

Autor

Institut

\section{G. Plewig}

Hautklinik der LMU München

\section{Bibliografie}

DOI http://dx.doi.org/

10.1055/s-0030-1256214 Akt Dermatol 2011; 37: 124-129 @ Georg Thieme Verlag KG Stuttgart · New York ISSN 0340-2541

\section{Korrespondenzadresse} Prof. Dr. med. Dr. h.c.

\section{Gerd Plewig}

Klinik und Poliklinik

für Dermatologie

LMU München

Frauenlobstr. 9-11

80337 München

Gerd.Plewig@

med.uni-muenchen.de

\section{Zusammenfassung \\ $\nabla$}

Johann Peter Frank (1745-1821) kam aus dem kleinen Ort Rodalben im Pfälzer Wald und machte als Arzt eine eindrucksvolle Karriere am Übergang des 18. zum 19. Jahrhundert. Wirkstätten waren Baden-Baden, Rastatt, Göttingen, Pavia, Wien, Wilna, St. Petersburg, und abermals Wien. Er gilt als Begründer der Sozialmedizin, des öffentlichen Gesundheitswesens, der Ordnung des Ärztestandes und der Apotheker, zugleich mit

Ist Johann Peter Frank vergessen von den Dermatologen unserer Tage? Ich gebe zu, dass sein Name mir unbekannt war bis zu dem Zeitpunkt, wo ich verschiedene Werke und Ausgaben seines umfassenden Oeuvres in den Händen hielt und mit viel Freude darin las.

Nicht vergessen ist er dem öffentlichen Gesundheitsdienst in Deutschland, vor allem durch die Aktivitäten der Johann-Peter-Frank-Gesellschaft mit Sitz in Rodalben. Diese Gesellschaft verleiht jährlich die Johann-Peter-Frank-Medaille an einzelne oder mehrere Persönlichkeiten, die sich um die öffentlichen Gesundheitsdienste verdient gemacht haben.

\section{Lebenslauf \\ $\nabla$}

Johann Peter Frank wurde am 19. März 1745 in Rodalben geboren. In der waldreichen Region des Pfälzer Waldes liegt der Ort zwischen Pirmasens und Kaiserslautern. Das im Jahr 1728 erbaute Fachwerkhaus, in dem er geboren wurde, ist liebevoll restauriert und erinnert an den großen Sohn der Gemeinde. 1761 beginnt Johann Peter Frank das Philosophiestudium in Metz und wird 1763 zum Dr. phil. promoviert. Seinen insgeheimen Wunsch, Medizin zu studieren, kann er bereits 1765 an den Universitäten Heidelberg und einem Einfluss auf die Dermatologie. Den Dermatologen ist er kaum bekannt geblieben, die Johann-Peter-Frank-Gesellschaft ehrt ihn jedoch durch die Vergabe einer Medaille für die Verdienste der öffentlichen Gesundheitsdienste. Zahlreiche mehrbändige Werke hat er verfasst, die in viele europäische Sprachen übersetzt wurden. Sein Sohn Joseph Frank (1771 - 1842), ebenfalls Mediziner, hat ein bedeutendes Buch über Hautkrankheiten geschrieben.

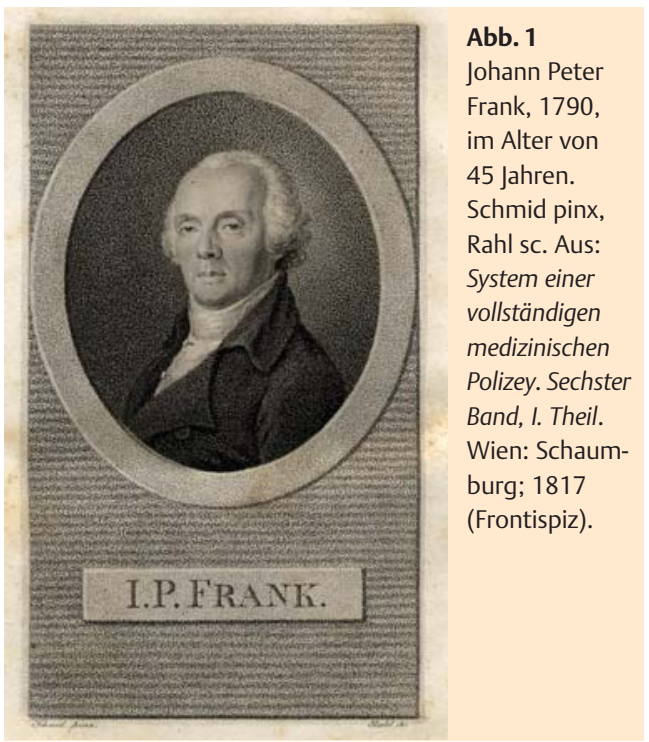

Straßburg umsetzen. Erhalten ist seine Dissertation De cunis infantum aus dem Jahr 1766. Um als Arzt in Baden-Baden praktizieren zu können, musste er, den damaligen politischen Verhältnissen entsprechend, das französische Examen nachholen, welches er 1767 an der Universität Pont-à-Mousson ablegt. Von nun an beginnt ein wechselvolles, ja auch rastloses Leben, zunächst als Arzt in Baden-Baden (1767), Garnisons- und 
Stadtarzt in Rastatt (1769) und als Landphysicus und Leibarzt des Fürstbischofs von Speyer (1772). Bereits 1784 nimmt er einen Ruf der Universität Göttingen als Professor der Medizinischen Klinik an. Ungewöhnlich für die damalige Zeit zieht es ihn in andere europäische Zentren und Metropolen. Einen besonders erfolgreichen Lebensabschnitt verbringt er seit 1786 in Pavia, in der herausgehobenen Position als Klinikdirektor und Generaldirektor für die Organisation des Medizinalwesens für die gesamte Lombardei. Mit großen Ehrerbietungen der Universität und der Bevölkerung verlässt Frank 1795 Pavia und folgt dem Ruf des Kaisers nach Wien. Die Wiener Militärakademie, bedingt durch die politisch-strategischen Operationen der Zeit, ist von außerordentlicher Bedeutung für das Herrscherhaus. So erhält Frank den Auftrag, den Militär-Sanitätsdienst grundlegend zu regeln. Als Direktor des Allgemeinen Krankenhauses bezieht er das formidable Gehalt von 5000 Gulden pro Jahr. Wie bereits in Pavia kann Frank sein Organisationstalent in Wien entfalten. Er führt die Pathologische Anatomie als Lehrfach ein und wird bald als beliebter akademischer Lehrer gefeiert. Allerdings bleiben Anfeindungen aus dem Fakultätskreis nicht aus, sodass er neun Jahre später 1804 dem Ruf nach Wilna (Vilnius, Litauen) als Professor der Klinik folgt. 1805 holt Zar Alexander ihn als Professor der Medizinisch-Chirurgischen Akademie nach St. Petersburg. Zugleich wird er Leibarzt der Zarenfamilie. 1808 kehrt er nach Wien zurück und erhält die glänzende Pension von 3000 Rubel vom Zar. In dieser Zeit wird der europäische Kontinent von Kriegen überzogen, und die Truppen Napoleons rücken in Wien ein. Die Historie berichtet, dass Kaiser Napoleon Johann Peter Frank in Wien konsultierte. Dabei lag der Kaiser entspannt in einer Badewanne und der „Greis“ Frank (damals 64 Jahre alt) musste während der langen Gespräche stehen. Napoleon bemühte sich, Frank als Leibarzt zu gewinnen und nach Paris zu holen. Intrigen aus dem Umfeld Napoleons verhinderten dieses jedoch, im Nachhinein ein glücklicher Umstand für Johann Peter Frank. Nach einer kurzen Zeit im Ruhestand in Freiburg im Breisgau (1810) geht er erneut nach Wien zurück (1811), wo er weiter als Arzt tätig ist. Am 24. April 1821 stirbt er. Sein Grab befindet sich im Ehrenfeld 32A, Nummer 3, auf dem Zentralfriedhof in Wien. Verbindet man die Stationen auf der europäischen Landkarte, die Frank zwischen Rodalben und Wien durchlaufen hat, entsteht ein weitreichendes Netz, womit der Vergleich als Wanderer zwischen den Welten verständlich wird.

\section{Das wissenschaftliche Werk \\ $\nabla$}

Glücklicherweise hat Johann Peter Frank ein umfassendes literarisches Werk hinterlassen. Mit großem Fleiß, ständiger Zielstrebigkeit und einer bis auf den heutigen Tag eindrucksvollen Vision entstehen verschiedene Hauptwerke, die vorwiegend sozialmedizinischen Themen, der Gesundheitsvorsorge, aber auch der Dermatologie gewidmet sind ( $\bullet$ Abb. 1-8).

Mit besonderem Interesse habe ich die sechs Bände sowie die Supplementbände von System einer vollständigen medicinischen Polizey gelesen ( $\bullet$ Abb. 1, 2).

\begin{tabular}{|c|c|}
\hline $\begin{array}{l}\text { System einer vollständigen } \\
\text { medicinischen Polizey }\end{array}$ & $\begin{array}{l}1779 \text { - } 1819, \text { Vol I-VI, Suppl Vol } \\
\text { in } 9 \text { Bänden } \\
\text { Mannheim, Tübingen, Wien }\end{array}$ \\
\hline $\begin{array}{l}\text { De curandis hominum } \\
\text { morbis epitome praelectionibus } \\
\text { academicis dictata }\end{array}$ & $\begin{array}{l}1792 \text { - } 1821, \text { Vol IX } \\
\text { Mannheim, Stuttgart, Wien } \\
\text { Deutsche, französische, italienische } \\
\text { Ausgaben, mehrfache Auflagen }\end{array}$ \\
\hline $\begin{array}{l}\text { Grundsätze über die Behand- } \\
\text { lungen der Krankheiten des } \\
\text { Menschen zu akademischen } \\
\text { Vorlesungen bestimmt }\end{array}$ & $\begin{array}{l}\text { 1794-1831, Vol IX in } 8 \text { Bänden } \\
\text { Mannheim }\end{array}$ \\
\hline
\end{tabular}

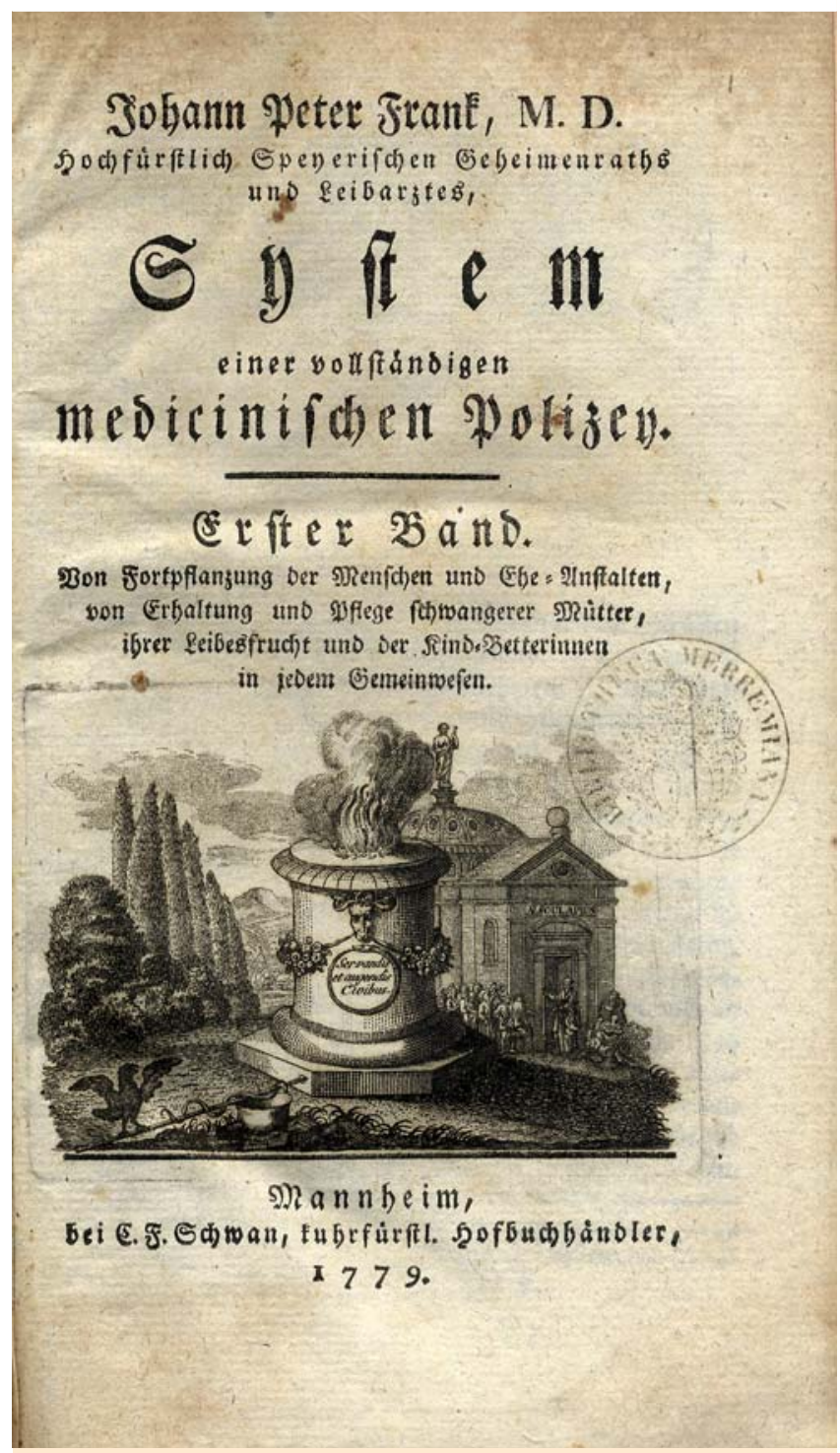

Abb. 2 Titelblatt mit Vignette aus: System einer vollständigen medizinischen Polizey. Erster Band. Mannheim: Schwan; 1779.

In $\odot$ Tab. 2 sind auszugsweise die Themen aufgeführt, um die Frank sich immer wieder bemühte. Das Wort „medicinische Polizey“ muss aus dem Sprachgebrauch des ausgehenden 18. und beginnenden 19. Jahrhunderts verstanden sein und ist nach heutigem Verständnis in die Sozialmedizin, öffentliche Gesundheitsfürsorge, Studienordnung und das Krankenhauswesen einzuordnen. Viele der Themen sind heute nicht mehr so aktuell, müssen jedoch im Kontext der damaligen Zeit gesehen werden. So war 
der Scheintod und die Befürchtung, bei lebendigem Leibe begraben zu werden, ein Thema der Zeit, wiederholt aufgegriffen von der Fachpresse und Laienwelt. Wer Angst hatte, zu früh, also vor Eintritt des wirklichen Todes, beerdigt zu werden, konnte sich eine Art Grabkammer bestellen, um beim Erlangen des Bewusstseins ein Signal an die „Oberwelt“ zu vermitteln. Technische Zeichnungen, wie das Alarmsystem funktionierte, finden sich in unterschiedlichen historischen Quellen.

Tab. 2 Gebiete, die im System einer vollständigen medicinischen Polizey abgehandelt werden.

\begin{tabular}{|c|ll|}
\hline Band & I & Sexualität, Zölibat, Ehe, Schwangerschaft \\
\hline II & Ledige Mütter, Abtreibung, Unfälle, Stillen, Schulen \\
\hline III & Nahrungsmittel, Getränke, Freizeit, Wohnen \\
\hline IV & Öffentliche Sicherheit, Wasser, Feuer, Tiere \\
V & $\begin{array}{l}\text { Scheintod, Beerdigung, Friedhof } \\
\text { VI }\end{array}$ & $\begin{array}{l}\text { Ausbildung der Ärzte, Barbiere, Bader, Tiermediziner, } \\
\text { Prüfungen }\end{array}$ \\
\hline Suppl & I- III & Apothekenordnung, Studienplan, Krankenhausbau \\
\hline
\end{tabular}

Ein anderes, ebenso umfassendes Werk entstand mit De curandis hominum morbis epitome praelectionibus ( $\triangle \mathbf{A b b} . \mathbf{3})$. Erscheinungsjahr, Verlagsorte und Verleger zeigen die Stationen, an denen Frank wirkte und beständig an seinen Büchern arbeitete. Mehrfache Auflagen erschienen, neben der deutschen auch eine französische und italienische $(\bullet$ Tab. 1$)$.

Zu seinem Sohn Joseph Frank (1771-1842) hatte Johann Peter Frank eine besondere Beziehung. Joseph arbeitete zeitweise für seinen Vater und folgte ihm im Amt als Leiter der medizinischen Einrichtung im Dienste des Zaren. Unter anderem verfasste er Die Hautkrankheiten. Ein Handbuch für Lehrer und Lernende, herausgegeben 1833, sowie Praxeos medicae universae praecepta. Der große Medizinhistoriker Richter gibt auf Seite 181 einen langen und sehr positiven Kommentar bezüglich der Verdienste um die Dermatologie, die leider von den modernen Historikern vergessen wird. So sollte neben Johann Peter auch sein Sohn Joseph Frank wieder in unser Geschichtsbewusstsein gebracht werden.

\section{Quellennachweise}

Bibliothekare und Antiquariatshändler sind unsere wichtigsten Partner, wenn es gilt, die Spuren der Vergangenheit zu lesen und zu deuten. In den Objektbeschreibungen finden sich für uns Dermatologen nicht immer verständliche Zitierungen, die aber nach einer Lernphase durchaus verständlich sind. Folgende Auswahl von Eintragungen finden sich zu Frank ( $\bullet$ Tab. 3).

Tab.3 Quellennachweis zu Johann Peter Frank.

Garrison/Morton, 1599
Von Engelhardt I, 186
Schönbauer: Das Medizinische Wien, 155-161
Eulner: Die Entwicklung der medizinischen Spezialfächer an den
Universitäten des deutschen Sprachgebietes, 141
Richter, 181
Hirsch/Hübotter II, 598 - 601
Gehört unstrittig zu den hervorragendsten Ärzten aller Zeiten
Unvergängliches Verdienst für öffentliche Gesundheitspflege
Umfang und Inhalt zu den bedeutendsten Werken, die je erschienen

D $E$

\section{CURANDIS HOMINUM MORBIS}

\author{
E $P$ P. I $T$ T $O$ O $M \quad M$
}

PRAELECTIONIBUS ACADEMICIS DICATA

\author{
$A U C T O R E$ \\ JOANNE PETRO FRANK
}

SAC. CAES. ET REG. AP. MAJ. A CONSILIIS

MAGISTRATUS POLITICI ET OECONOM. MEDIOLAN. FACULTATIS MEDICAE AC NOSOCOMIORUM PER INSUBRIAM AUSTR. PRAESIDE, XENODOCHII PAPIENS. AD S. MATTH. MODERATORE, THERAPIAE SPEC. ET CLINICES IN TICINENSI ACADEMIA PROFESSORE P. ET O. ACADEMIAR. REG. SCIENTIAR. GOETTING. MANTUANAE, ET ELECT. MOGUNT. TUM SOCIET. PATRIOT. MEDIOL. ET MEDIC. HELVET SODALI -

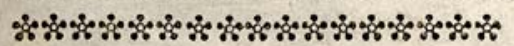

EDITIO PRIMA.

$L I B$ E R III.

DE EXANTHEMATIBUS.

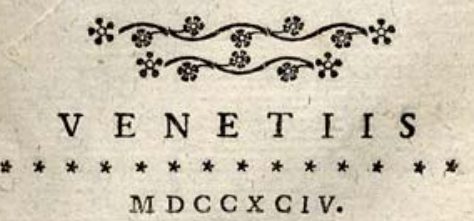

Abb. 3 Titelblatt aus: De curandis hominum morbis epitome praelectionibus academicis dicatata. Liber III. De exanthematibus. Editio prima. Venetiis, ohne Verlagsangabe, 1794.

\section{Autobiografie und Biografien}

Im Alter von 57 Jahren, also noch nicht am Ende seines schaffensreichen Lebens, gibt Johann Peter Frank eine 174-seitige Autobiografie heraus Biographie des D. Johann Peter Frank von ihm selbst geschrieben, verlegt bei Schaumburg in Wien 1802. Es folgen 1802 eine italienische und posthum 1948 eine englische Ausgabe. Professor Dr. med. Dr. phil. Erna Lesky (1911 - 1986), die bedeutende Medizinhistorikerin, von 1957 bis 1979 Leiterin des Instituts für Geschichte der Medizin an der Universität Wien, - also auf geschichtsträchtigem Grund und der langjährigen geografischen Nachbarschaft von Johann Peter Frank -, gab das Buch zu Frank heraus: Johann Peter Frank, seine Selbstbiographie.

Die Johann-Peter-Frank-Gesellschaft hat eine sehr umfassende Dokumentation zu Johann Peter Frank, seiner Familiengeschichte, seinem Lebenslauf, seinem literarischen Verzeichnis und der bis in unsere Tage reichenden Aufarbeitung dieses großen Mediziners herausgegeben. 


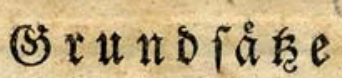 úber bie Ze Der Sirantbeiten bes s)enteden, $x$ \\ afabemifden 30 clefungen beftim Bon \\ Sobann \$Petet FtanE,}

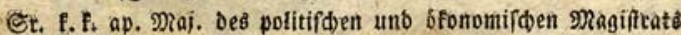

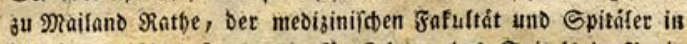

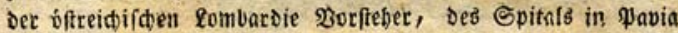
jum beil. Matbias Dbernuffeher, Det befondern Szeilfunde und SRinif auf Der univerfitát zu pavia oro. iff. Lebret, verfóbiedes ner selebrten Befellfonften Mitgfiede.

Erfter Theil, won Den Siebern.

Unter eignet 2(ufficht Des Saertn Werfaffers aus bem Eateinifchen uberfest.

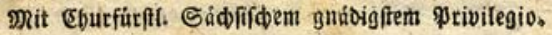

M a n n

bei डdwan und હु⿰氵, I794.

Abb. 4 Titelblatt aus: Grundsätze über die Behandlung der Krankheiten des Menschen zu akademischen Vorlesungen bestimmt. Erster Theil, von den Fiebern. Mannheim: Schwan und Götz; 1794.

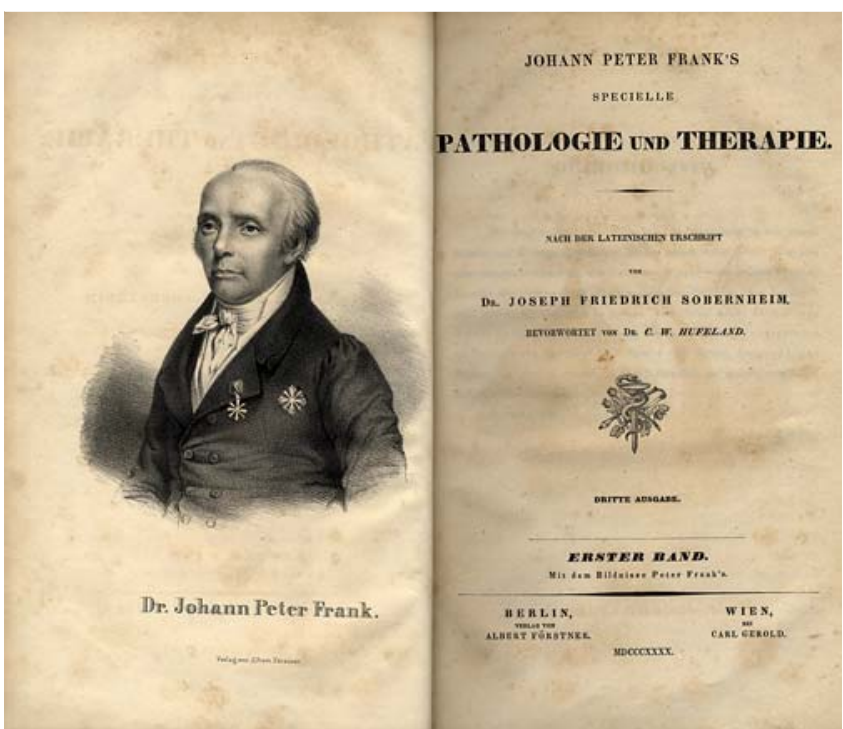

Abb. 5 Johann Peter Frank (Frontispiz), und Titelblatt aus: Specielle Pathologie und Therapie. Dritte Ausgabe. Wien: Förstner, Berlin \& Gerold; 1840.

\section{Gedenktafeln, Denkmäler, Büsten, Medaillen}

Wie hoch die Wertschätzung von Vater und Sohn Frank war und bis auf den heutigen Tag ist, zeigt eine Auswahl von Ehrungen, die sich entlang des Lebensweges finden ( $\bullet$ Tab. 4).

Tab.4 Gedenktafeln, Denkmäler, Büsten und Medaillen.

\begin{tabular}{|c|c|}
\hline Büste und Gedenktafel & Aula Scarpa, Teatro Anatomica, Pavia \\
\hline Gedenktafel & Im Hof der Universität Pavia \\
\hline Denkmal & $\begin{array}{l}\text { Johann Peter und Johann Frank, } \\
\text { Universität Pavia } \\
\text { Wiener Allgemeines Krankenhaus }\end{array}$ \\
\hline Denkmal und Gedenktafel & Geburtshaus Rodalben \\
\hline Flachrelief & Universität Wilna \\
\hline Büste & $\begin{array}{l}\text { Österreichische Bundesregierung für } \\
\text { Deutsches Hygiene-Museum Dresden } \\
\text { Rodalben, Museum }\end{array}$ \\
\hline Gedenkmünzen & $\begin{array}{l}\text { Johann Peter Frank } \\
\text { Johann Peter und Johann Frank }\end{array}$ \\
\hline Straßennamen & $\begin{array}{l}\text { Rodalben, Bruchsal, Pavia (Via Guiseppe } \\
\text { Frank), Wien (Frankengasse) }\end{array}$ \\
\hline
\end{tabular}

Das Deutsche Hygiene-Museum in Dresden besitzt Büsten von ausgewählten großen Ärzten des Abendlandes ( Tab.5). Auftakt zur Aufstellung der Büsten war 1931 mit einem Geschenk der Österreichischen Staatsregierung. Die Bildhauerin Cäcilie Danzer-Rüstler schuf 1930 eine Marmorbüste von Johann Peter Frank. Diese Büste befindet sich heute als Leihgabe in Rodalben. Der Ort Rodalben ehrt in besonderer Weise Johann Peter Frank. Man hat Straße und Platz nach ihm benannt, ein Museum installiert, sechs Skulpturen des Künstlers Stephan Müller in das Stadtbild gestellt und eine Bronzebüste des Bildhauers Karl Nuding fertigen lassen.

Tab. 5 Büsten im Deutschen Hygiene-Museum.

\begin{tabular}{|c|c|c|}
\hline Frank & 1931 & Stiftung der österreichischen Staatsregierung \\
\hline Hippokrates & 1931 & Stiftung der griechischen Regierung \\
\hline Koch & 1932 & Stiftung der sächsischen Staatsregierung \\
\hline Pettenkofer & 1932 & Stiftung Dr. Poppe \\
\hline Virchow & 1932 & Stiftung der Stadt Berlin \\
\hline Paracelsus & 1938 & $\begin{array}{l}\text { Stiftung der Deutschen Paracelsus-Gesell- } \\
\text { schaft }\end{array}$ \\
\hline Behring & 1942 & Stiftung Behring-Werke \\
\hline Pasteur & 1947 & Stiftung der französischen Regierung \\
\hline
\end{tabular}

\section{Danksagung}

Oberamtsrat a.D. Alois Dauenhauer, geboren 1929 in Rodalben, Mitglied des Landtags Rheinland-Pfalz 1975-1991, Bürgermeister der Stadt Rodalben (1982-1994), derzeitiger Vorsitzender der Johann-Peter-Frank-Gesellschaft, leistete wertvolle Unterstützung für Quellen und Dokumente. 


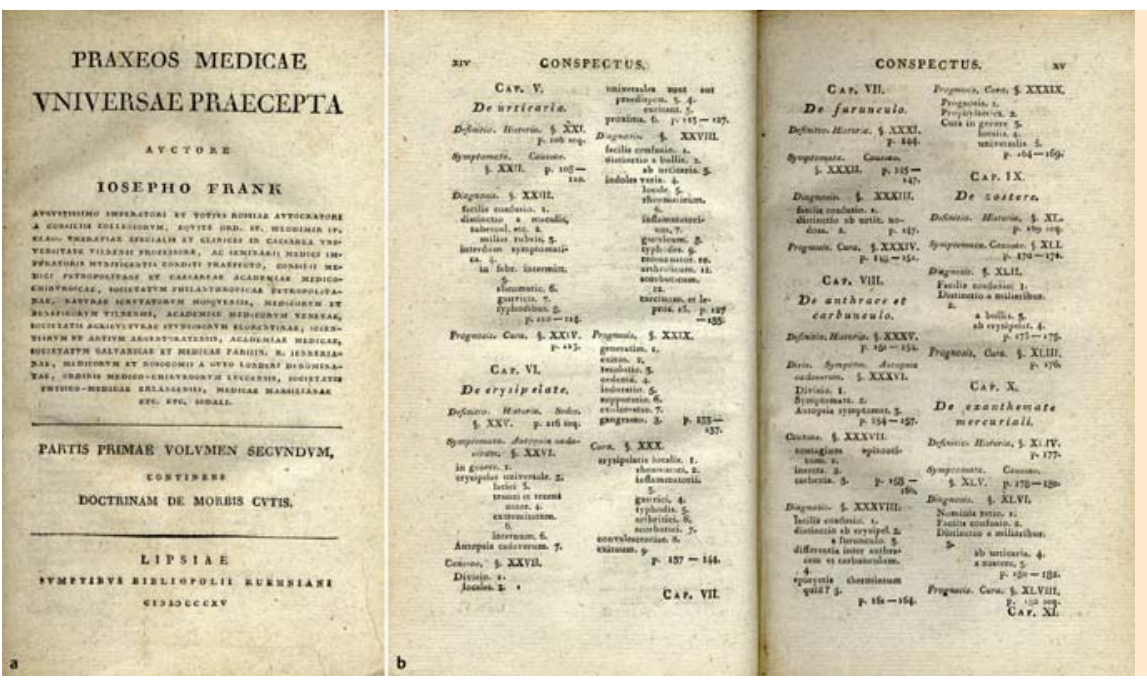

Abb. 6 Joseph Frank. a Titelblatt aus: Praxeos medicae universae praecepta. Partis primae volumen secundum, continens Doctrinan de morbis cutis. Lipsiae: Kuehniani; 1815. b Inhaltsangabe (Auszug).

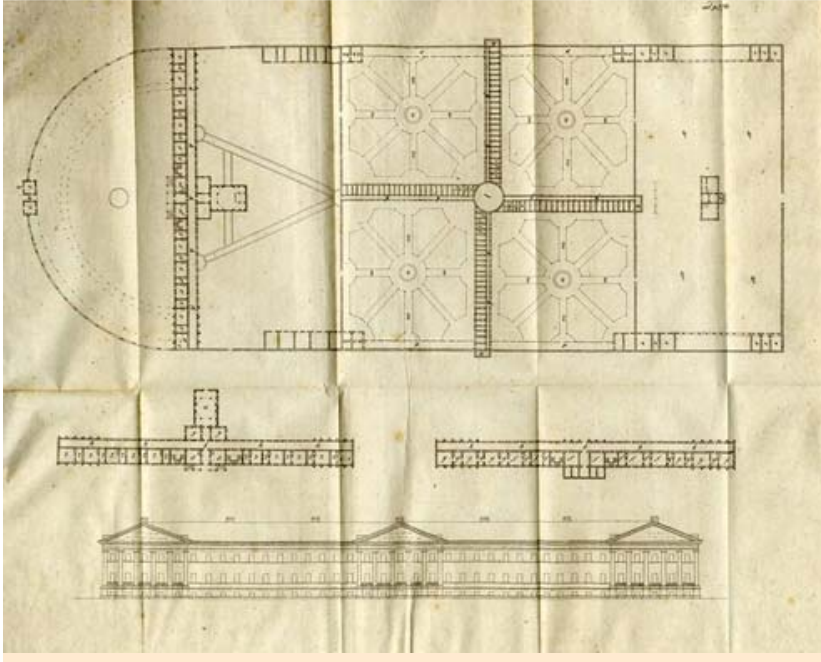

Abb. 7 Joseph Frank. Gravierte Architekturzeichnung für einen modernen Krankenhausbau und patientengerechte Unterbringung. Aus: Praxeos medicae universae praecepta. Partis secundae volumen primum, sectio prima. De maniis pp 690ff. Falttafel zur Seite 778. Lipsiae: Kuehniani; 1818.

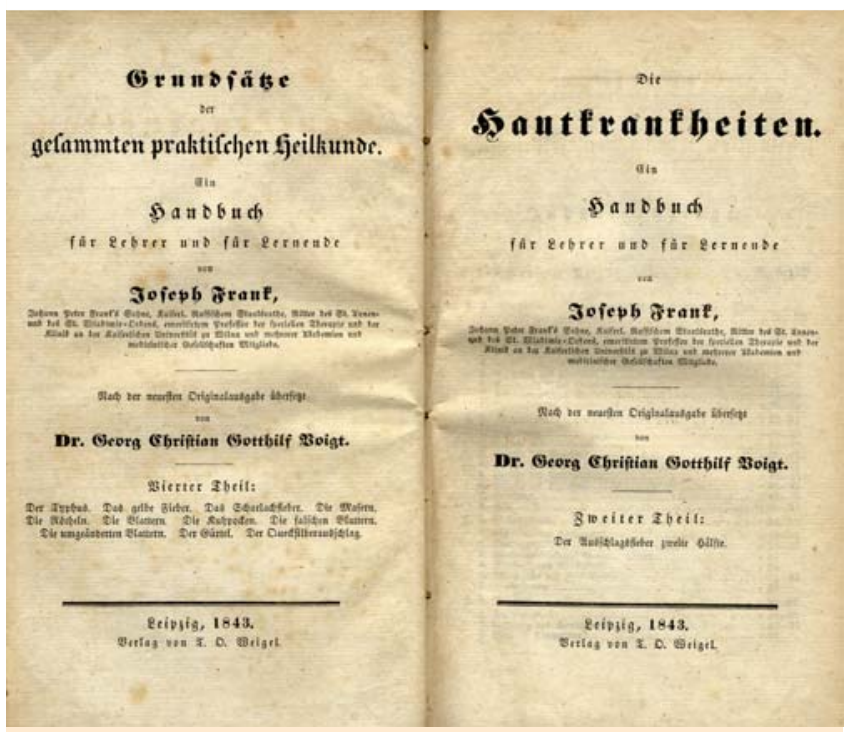

Abb. 8 Joseph Frank. Die Hautkrankheiten. Ein Handbuch für Lehrer und für Lernende. Zwei Teile in einem Band. Leipzig: Weigel; 1843.

\section{Abstract}

\section{Johann Peter Frank (1745-1821). Migrant through Europe, Founder of Social Medicine, Public Health, with an Impact on Dermatology}

Johann Peter Frank (1745-1821) was born in Rodalben, a small town in the Palatinate Forest (Pfälzer Wald). He had an impressive career as a physician at the turn of the $18^{\text {th }}$ to the $19^{\text {th }}$ century. Stations of his appointments were Baden-Baden, Rastatt, Göttingen, Pavia, Vienna, Wilna (Vilnius), St. Petersburg, and again Vienna. Frank is considered the founder of social medicine and public health, also structuring the organisations of doctors and apothecaries. Finally, he had an impact on dermatology. He seems to be forgotten in dermatology, however, the Johann-Peter-Frank-Gesellschaft (Society) honours persons with a medal for their achievement in public health. Frank wrote many books which were translated into several European languages.

His son Josef Frank (1771 - 1842), also a physician, published a respectable book on dermatology.

\section{Literatur}

1 Dauenhauer A, Mattheis H. Johann Peter Frank (1745-1821). Gegen Armut und Krankheit. Leben und Wirken eines großen Arztes. Dokumentation aus Texten, Urkunden und Bildern. Rodalben: Johann-Peter-Frank-Gesellschaft; 2007

2 Eulner $\mathrm{H}-\mathrm{H}$. Die Entwicklung der medizinischen Spezialfächer an den Universitäten des deutschen Sprachgebietes. Stuttgart: Enke; 1970

3 Frank J. Die Hautkrankheiten. Ein Handbuch für Lehrer und für Lernende. Zweiter Theil: Der Ausschlagsfieber zweite Hälfte. Vierter Theil: Der Typhus. Das gelbe Fieber. Das Scharlachfieber. Die Masern. Die Rötheln. Die Blattern. Die Kuhpocken. Die falschen Blattern. Die umgeänderten Blattern. Der Gürtel. Der Quecksilberausschlag. Nach der neueste Originalausgabe übersetzt von Dr. Georg Christian Gotthilf Voigt. Leipzig: Weigel; 1843

4 Frank J. Praxeos medicae universae praecepta. Vol I,1 Prolegomena, doctrinam de febribus atque de inflammationibus generalem 1811. Vol I,2 Doctrinam de morbis cutis 1815 . Vol II,1,1 Doctrinam de morbis systematis nervosi in genere, et de iis cerebri in specie cum tabula aena 1818. Vol II,1,2 Doctrinam de morbis columnae vertebralis, singulorum nervorum aliisque ex variis systematis nervosi partibus ortum habentibus nec non oculorum, aurium, narium et cavitatum nasalium 1821. Vol II,2,1 Doctrinam de morbis laryngis, tracheae et corporis thyreoidei, thecae thoracicae, pleurae, mediastini, thymi et pulmorum. Lipsiae: Kuehniani; 1823 
5 Frank JP. De educatione infantum. Dissertatio inauguralis. Umbearbeitung durch Professor Gattenhof und Herausgabe unter dem Titel: De cunis infantum. Universität Heidelberg; 1766

6 Frank JP. De curandis hominum morbis epitome praelectionibus academicis dicata. Liber I, De febribus 1792. Liber II, De inflammationibus 1792. Liber III, De exanthematibus 1792. Liber IV, De impetiginibus 1793. Liber V, De profluviis 1794. Liber VI, De profluviis 1807. Mannheim: Schwan; 1794

7 Frank JP. Abhandlung von Eröffnung schwangerer Mütter, welche unentbunden gestorben sind, und zur Rettung ihrer Leibesfrüchte. Zur Nachahmung für Seelsorger, Beamte und Hebammen des Hochstifts Fulda. Stahelische Schriften, 1801

8 Frank JP. Biografie des Johann Peter Frank. Wien: Schaumburg; 1802

9 Frank JP. Grundsätze über die Behandlung der Krankheiten des Menschen, zu akademischen Vorlesungen bestimmt; unter eigener Aufsicht des Herrn Verfassers aus dem Lateinischen übersetzt und von ihm selbst revidiert. Erster Theil, Von den Fiebern, Mannheim: Schwan und Götz; 1794. Zweyter Theil, Von den Entzündungen, Mannheim; 1795. Dritter Theil, Von den akuten Hautausschlägen, Mannheim; 1795. Vierter Theil, Von den Ausflüssen, Mannheim; 1797. Fünfter Theil, Von den Ausflüssen, Mannheim; 1797. Sechster Theil, Von den Ausflüssen, Mannheim; 1807. Siebenter Theil, Von den Zurückhaltungen. Stuttgart: 1811

10 Frank JP. Johann Peter Frank's specielle Pathologie und Therapie. Nach der lateinischen Urschrift des Dr. Joseph Friedrich Sobernheim. Befor- wortet von Dr. C.W. Hufeland. Dritte Ausgabe. Förtner, Berlin \& Gerold, Wien; 1840. Erster Band. Mit dem Bildnisse Peter Frank's. Zweiter Band 1841

11 Frank JP. System einer vollständigen medicinischen Polizey. Vol I, Mannheim: Schwan; 1779. Vol II, Mannheim: Schwan; 1780. Vol III, Mannheim: Schwan; 1783. Vol IV, Mannheim: Schwan; 1788. Vol V, Tübingen: Cotta; 1813. Vol VI,1 - 2, Wien: Schaumburg; 1817. Vol VI,3, Wien: Schaumburg; 1919. Suppl I, Tübingen: Cotta; 1812. Suppl II Leipzig: Kühn; 1825. Suppl III, Leipzig: Kühn; 1825

12 Hirsch A, Hübotter F. Biographisches Lexikon der hervorragenden Ärzte aller Zeiten und Völker. Zweiter Band. München: Urban \& Schwarzenberg; 1962

13 Lesky E. Johann Peter Frank, seine Selbstbiographie. Herausgegeben, eingeleitet und mit Erläuterungen versehen von Professor Erna Lesky. Hubers Klassiker der Medizin. Vol 12, Bern: Huber; 1969

14 Norman JM, ed. Morton's medical bibliography. An annotated checklist of texts illustrating the history of medicine (Garrison and Morton). 5th ed. Brookfield: Scolar Press; 1991

15 Richter P. Geschichte der Dermatologie. In: Jadassohn J, Hrsg. Handbuch der Haut- und Geschlechtskrankheiten. Berlin: Springer, XIV/2; 1928

16 Schönbauer L. Das medizinische Wien. Geschichte, Werden, Würdigung. Berlin: Urban \& Schwarzenberg; 1944

17 Engelhardt D von, Hrsg. Biographische Enzyklopädie deutschsprachiger Mediziner. München: Sauer, I/A-Q; 2002 